\title{
ESTIMATES FOR THE SZEGÖ AND POISSON KERNELS OF SUFFICIENTLY ROUNDED TUBE DOMAINS
}

\author{
BY LAWRENCE J. DICKSON ${ }^{1}$ \\ Communicated by Alberto Calderón, February 28, 1972
}

In this paper we obtain estimates for the decrease at infinity of the Szegö and Poisson kernels, $S_{\Gamma}(X, Y)=S_{\Gamma ; X}(Y)$ and $P_{\Gamma}(X, Y)=P_{\Gamma ; X}(Y)$ $=\left|S_{\Gamma}(X, Y)\right|^{2}\left\|S_{\Gamma ; X}\right\|_{2}^{-2}$, associated with proper cones $\Gamma \subset R^{n}$ which are sufficiently smooth and satisfy certain curvature conditions. These estimates verify, for these cases, the conjecture of Stein (see [2], [4]) that the Poisson integral of an $L^{1}$ function converges restrictedly almost everywhere to that function on the distinguished boundary of a tube domain (Corollary IA). These and other results about the Poisson kernel will be elaborated on in [1].

Let $\Gamma$ be a proper cone of $\boldsymbol{R}^{n}$ (that is, a nonempty, open, convex cone whose closure contains no whole line), $\Gamma^{*}$ its dual cone

$$
\Gamma^{*}=\left\{Y \in R^{n}:(X, Y)>0 \forall X \in \bar{\Gamma}-\{0\}\right\},
$$

which is also proper, and $\Omega=\Omega_{\Gamma}$ its tube domain

$$
\Omega=\Gamma \times i R^{n}=\left\{Z \in C^{n}: \operatorname{Re}(Z) \in \Gamma\right\} .
$$

For $X \in \Gamma$ define the nonempty compact section $C_{\Gamma^{*} ; X}=C_{X}^{*}$ of $\bar{\Gamma}^{*}$ as follows:

$$
C_{X}^{*}=\left\{Y \in \bar{\Gamma}^{*}:(X, Y)=1\right\} \subset\{Y:(X, Y)=1\} \approx R^{n-1} ;
$$

and similarly for $C_{\Gamma ; Y}=C_{Y}, Y \in \Gamma^{*}$.

We will say $\Gamma$ is $C^{N}, N \geqq 0$, if $\partial C_{Y}$ is $C^{N}$. $\Gamma$ will be said to satisfy the "flat curvature condition" if for some proper circular cone $\Delta$ of $\boldsymbol{R}^{n}$ and every $P \in \partial \Gamma$ there is a rotation $\rho_{P}$ of $R^{n}$ such that $P \in \partial\left(\rho_{P} \Delta\right)$ and $\rho_{P} \Delta \subset \Gamma$. The dual condition, the "sharp curvature condition," is stated similarly but reverses the last inclusion. We exclude, in our theorems, the trivial cases $n=1,2$.

THEOREM I. Suppose $\Gamma$ is a proper cone of $\boldsymbol{R}^{n}$, where

(a) $n=3$ and $\Gamma$ satisfies the flat curvature condition, or

(b) $n \geqq 4, \Gamma$ is $C^{[n / 2]}$, and $\Gamma$ satisfies the sharp curvature condition.

AMS 1970 subject classifications. Primary 31B10, 31B25; Secondary 32F05, 52A20.

Key words and phrases. Szegö and Poisson kernels, proper cones, compact section, decrease at infinity, contour integration, maximal function.

1 This research was partially supported by an NSF Graduate Fellowship.

Copyright (C) American Mathematical Society 1972 
Then, if we define $m_{\Gamma}$ on $\boldsymbol{R}^{n}$ by

$$
\begin{aligned}
m_{\Gamma}(Y) & =1 \quad \text { if }|Y| \leqq 1, \\
& =|Y|^{-n / 2}\{\max [1, \operatorname{dist}(Y, \partial \Gamma \cup-\partial \Gamma)]\}^{-1 / 2} \quad \text { if }|Y| \geqq 1,
\end{aligned}
$$

there exists for every compact $S \subset \Gamma$ a constant $k=k_{S}<\infty$ such that

$$
\left|S_{\Gamma ; X}\right| \leqq k m_{\Gamma} \quad \forall X \in S,
$$

and hence $k_{2}=k_{2}(S)<\infty$ such that

$$
P_{\Gamma ; X} \leqq k_{2} m_{\Gamma}^{2} \quad \forall X \in S .
$$

COROLlaRY IA. If $\Gamma$ satisfies the conditions of Theorem I and $f \in L^{1}\left(R^{n}\right)$, and we define the Poisson integral of $f$ by

$$
P f(X+i Y)=\left(P_{\Gamma ; X} * f\right)(Y), \quad X \in \Gamma, Y \in R^{n},
$$

then for almost every $Y_{0} \in R^{n}, P f(Z) \rightarrow f\left(Y_{0}\right)$ as $Z$ converges to $i Y_{0}$ restrictedly in $\Omega_{\Gamma}$.

(We say $Z \rightarrow Z_{0}$ restrictedly in $\Omega$ if $\{Z\} \subset \Omega, Z_{0} \in \bar{\Omega}, Z \rightarrow Z_{0}$, and for some $\delta>0, \operatorname{dist}(Z, \partial \Omega) \geqq \delta\left|Z-Z_{0}\right| \forall Z$.)

With a little more smoothness we attain an estimate which is the best possible, even for a circular cone (see [4]):

THEOREM II. Suppose $n \geqq 3$ and $\Gamma$ is a $C^{n}$ proper cone of $\boldsymbol{R}^{n}$ satisfying the sharp curvature condition. Then the conclusions of Theorem I hold with $m_{\Gamma}(Y)$ replaced by

$$
\begin{aligned}
\mu_{\Gamma}(Y) & =1 \quad \text { if }|Y| \leqq 1 \\
& =|Y|^{-n / 2}\{\max [1, \operatorname{dist}(Y, \partial \Gamma \cup-\partial \Gamma)]\}^{-n / 2} \quad \text { if }|Y| \geqq 1 .
\end{aligned}
$$

SKETCH OF PROOFS. Since every $C^{2}$ proper cone satisfies the flat curvature condition, the hypotheses of Theorem I imply $\Gamma^{*}$ satisfies the sharp curvature condition and is $C^{[n / 2]}$ if $n \geqq 4$, while those of Theorem II imply $\Gamma^{*}$ satisfies the sharp curvature condition and is $C^{n}$. These imply similar conditions for the $C_{X}^{*}$, uniformly for $X \in S$.

The crucial step is the radial integration of the usual formula for the Szegö kernel, which (see [2]) gives, for $S_{X}=S_{\Gamma ; X}$,

$$
S_{X}(Y)=\frac{(n-1) !}{(2 \pi)^{n}|X|} \int_{C_{*}^{*}}(1+i(Y, \sigma))^{-n} d \sigma,
$$

where $d \sigma$ is induced Lebesgue measure on the affine hyperplane $\{\sigma:(X, \sigma)=1\}$. If we set $K(X)=(n-1) ! /(2 \pi)^{n}|X|$ we get that, for $p \neq 0$,

$$
S_{X}(p Y)=\frac{K(X)}{p^{n}} \int_{C_{X}^{*}}(1 / p+i(Y, \sigma))^{-n} d \sigma .
$$


In Theorems I and II, the estimate is immediate for $|Y| \leqq 1$; thus, in (10), we may take $|Y|=1$ and $p \geqq 1$. Then a gross estimate, using only the sharp curvature condition for $\Gamma^{*}$, yields the estimate of Theorem II for $Y \in \bar{\Gamma} \cup-\bar{\Gamma}$. For the case $p \geqq 1,|Y|=1, Y \notin \Gamma \cup-\Gamma$, we use Fubini's theorem on (10) to get

$$
S_{X}(p Y)=\frac{K_{2}}{p^{n}} \int_{a}^{b}(1 / p+i y)^{-n} \theta(y) d y
$$

where $K_{2}=K_{2}(X, Y), a=a(X, Y)$, and $b=b(X, Y)$ are continuous, $a<0$ $<b,[a, b]=\left(Y, C_{X}^{*}\right)$, and $\theta(y)=\theta(X, Y, y)=\lambda^{n-2}\left(\left\{\sigma \in C_{X}^{*}:(Y, \sigma)=y\right\}\right)$ is smooth on $(a, b)$ to the same degree as $\Gamma^{*}$, continuously in $X$ and $Y$. $\lambda^{n-2}$ is induced Lebesgue measure.

The results of Theorem I and II, and intermediate estimates for intermediate degrees of smoothness, now follow straightforwardly (except for some complications for $|a|$ or $|b|$ small) if we approximate $\theta$ at $y=0$ by a polynomial $\widetilde{\theta}$, do a contour integration on the analytic integral with the contour passing below the origin, and estimate the remainder term crudely. The hypothesis " $C{ }^{N}$ " could, for greatest generality, be replaced by "uniformly Lipschitz $N-1$ order derivatives", which explains the apparent anomaly of Theorem I(a).

Corollary IA follows in the usual manner, by showing that the maximal operator $M=M_{\{Z\}}$ sending $f$ into $M f(Y)=\sup _{\{Z\}}\{|P f(Z+i Y)|\}$ is weak-type $(1,1)$ if $\exists \delta>0$ such that $Z \in\{Z\} \Rightarrow \operatorname{dist}\left(Z, C^{n}-\Omega\right)>\delta|Z|$. It suffices to show $\widetilde{M}$ is weak-type $(1,1)$, where $\tilde{M} f(Y)$ $=\sup _{\delta>0}\left(m_{\Gamma ; \delta}^{2} *|f|\right)(Y)$ and $m_{\Gamma \delta}^{2}(Y)=\delta^{-n} m_{\Gamma}^{2}(Y / \delta)$. This result follows, as in [2], by majorizing $m_{\Gamma}^{2}$ in the natural fashion by a sum of multiples of characteristic functions of rectangles centered at 0 , and applying 2.3 of [5].

\section{BIBLIOGRAPHY}

1. L. J. Dickson, Limit properties of Poisson kernels of tube domains (to appear).

2. Some limit properties of Poisson integrals and holomorphic functions on tube domains, Doctoral Dissertation, Princeton University, Princeton, N.J., 1971.

3. S. Karlin and L. S. Shapley, Geometry of moment spaces, Mem. Amer. Math. Soc. No. 12 (1953), 93 pp. MR 15, 512.

4. E. M. Stein, G. Weiss and M. Weiss, $H^{p}$ classes of holomorphic functions in tube domains, Proc. Nat. Acad. Sci. U.S.A. 52 (1964), 1035-1039. MR 31 \#3634.

5. E. M. Stein and N. J. Weiss, On the convergence of Poisson integrals, Trans. Amer. Math. Soc. 140 (1969), 35-54. MR 39 \#3024.

DePartment OF MATHEMATICS, University OF WAShington, SEATtLe, WaShington 98105 